\title{
Reducing Energy Consumption by Using Smart Metering Intelligent Systems
}

\author{
Ivaylo Atanasov, Anastas Nikolov, Evelina Pencheva \\ Faculty of Telecommunications, Technical University of Sofia, Sofia, 1756 Bulgaria \\ Emails: iia@tu-sofia.bgrnikolov.anastas@gmail.com enp@tu-sofia.bg
}

Abstract: Smart metering is aimed at efficient energy management. Its potential may be revealed using recent advances in machine type communications. This paper presents an approach to design web services for residential power control with prepaid functionality. The reduction in energy consumption is estimated for typical households applying heating control.

Keywords: Smart metering, heating/cooling control, payment management, resource structure, RESTful web services.

\section{Introduction}

Growing energy needs and exhaustion of energy sources call for usage of mechanisms that optimize energy consumption and employment of new smart technologies to facilitate the network control of distribution grids. Sustainable business models that guarantee improvements of energy efficiency incorporate information and communication technologies into the whole value chain of energy production, supply and consumption. Smart metering appears to be such an advanced technology which is concerned with gathering, evaluating and aggregating consumption and usage data, monitoring of operating status executed in real time, and sending of control signals. Smart metering is designed to provide customers with information on real-time basis about their domestic energy consumption [1].

Smart metering is one of the main application areas of Machine to Machine (M2M) communications. M2M communications establish conditions that allow individual devices to exchange bidirectional information with business application via a telecommunication network (wireline, wireless, private, public) [2]. M2M applications for smart metering can contribute to customer engagement in the process of energy saving by providing information about actual consumption and controlling individual devices within the home/building. For example, M2M enables smart control on heating, ventilation and air conditioning systems, which 
play an essential role in ensuring a pleasant, comfortable and safe home and work environment, but consume large amounts of energy [3, 4]. In [5], the authors propose model of predictive control on heating and cooling in a simple house which takes into account the current thermal conditions and the weather forecast. A model-based approach to design efficient control architecture for radiant heating/cooling systems aimed at increasing both thermal comfort for building occupants and energy saving is described in [6]. In [7], the authors consider home's occupancy and sleep patterns as well as outdoor temperature and user's preferences to apply intelligent energy control that improves energy efficiency for both heating and cooling spaces. An integrated approach to energy/power management and control of heating, ventilation and air conditioning system in a grid-connected microgrid with energy storage is suggested in [8]. In [9], a smart home automation system is designed which can be controlled in two different ways either by any internet-connected device or an operator panel.

Smart meters with prepayment functionality enable new and more flexible ways of meter topping up, and remote credit top-up facilities. Smart metering system allows the customer to prepay for usage by suitable payment means, to connect a supply and disconnect it when predetermined energy usage thresholds are reached [10-12]. Prepaid energy metering can be used to detect power thefts [13-15].

The variety of vertical solutions oriented to serve specific needs calls for reusable generic functionality that may be shared by different applications. This functionality has to be exposed by application programming interfaces.

European Telecommunications Standard Institute (ETSI) defines high level M2M system architecture that provides an overview of the components of a system, as well as the relationship between the individual components [16]. In order to allow for an extendable and flexible structure, generic M2M functions are defined as service capabilities. For M2M communications, REST (REpresentational State Transfer) architecture style is adopted. The main concept in REST is that a distributed application is composed of resources. The resource has a particular state that may be manipulated by four interactions: CREATE, UPDATE, DELETE and RETRIEVE called "verbs". REST relies heavily on HTTP, where resources are web-accessible. Each resource in REST can be addressed using unique HTTP URI (Uniform Resource Identifier).

RESTful web services appear to be key enabling technology for integrating smart devices in constrained environments with the web [17, 18]. In [19], the authors propose interoperability framework with several home devices to demonstrate the applicability of web service technology in heterogeneous environment. A framework, which systematically creates and manages contextaware service using various web services (e.g., information services, sensor services, networked appliances) is proposed in [20]. In [21], the authors propose M2M architecture that allows real time interaction between mobile clients and smart things (sensors and actuators) via a wireless gateway using RESTful web services. 
The survey on current research on web services for smart metering exposes either high level architectural principles or specific implementations. As far as our knowledge, there is no structural approach to RESTful web services design that follows existing standards for definition of resource structure. The aim of the current research is to develop a low level method for design of RESTful web services which may be used by different M2M applications for prepaid power control of electrical appliances. We provide an information model of resources representing smart metering with prepayment functionality and home appliance power control. Tree structures modelling relationship of different resource types are synthesized.

In the following sections, we present the idea behind the power control of electrical appliances as a kind of demand-response mechanism and evaluate the potential of prepaid functionality for energy usage monitoring. The second section starts with highlighting the potential of power control on electrical appliances, then the electrical appliance resources are modelled by tree structures, and finally the web service interfaces for heating/cooling control are described. Third section starts with identification of requirements to energy usage monitoring control and continues with definitions of managed objects for credit/prepayment options and multiple rate tariffs. The fourth section outlines the novelty and provides an estimation of the potential of residential heating/cooling control in a city. In the conclusion, we summarize our contributions.

\section{Energy management at home and buildings}

\subsection{Potential of power control on electrical appliances}

Smart metering may be used for secured communication with home and building energy management systems to remotely control the power of electrical appliances used for heating and cooling. It reflects the tolerance in temperature comfort of the householders. The idea is to reduce/increase the inside temperature by a small amount $\delta$. For a family house, the day temperature changes $\delta$ may vary as follows: $-1^{\circ} \mathrm{C}$ (in winter) or $+1^{\circ} \mathrm{C}$ (in summer) when the children are at school, $-2^{\circ} \mathrm{C}$ (in winter) or $+2^{\circ} \mathrm{C}$ (in summer) when everybody is sleeping, etc.

\subsection{Electrical appliance as a resource}

In order to control remotely an electrical appliance by means of RESTful web services, the electrical appliance needs to be considered as a resource with a state. The resource handling takes place by manipulating the air conditioner's states. Fig.1 shows the state model of an air conditioner.

An air conditioner may be controlled remotely only of it is powered on. The air conditioner moves from Unpowered state to Standby state by manual operation powerOn. When the air conditioner is in Standby state, it is ready to receive instructions. The transition from Standby state is triggered by the goToRef operation which instructs the air conditioner to heat/cool to the reference temperature. The air conditioner is in GoingToRef state, while it consumes energy to reach the reference temperature. When the referenced temperature is reached, the air conditioner 
reports the event (refTempReached notification) and it moves to SteadyRef state. In SteadyRef state, the air conditioner consumes energy to maintain the referenced temperature. In the beginning of the cool-off/heat-off period the goToEcon operation instructs the air conditioner to switch off its heating/cooling system in order to cool/heat to the economic temperature. This causes the transition to GoingToEcon state in which no energy is consumed. When the economic temperature is reached, the air conditioner reports the event (econTempReached notification) and moves to SteadyEcon state. In SteadyEcon state, the air conditioner consumes less energy to maintain the economic temperature than in SteadyRef state. At the end of the cool-off/heat-off period, the air conditioner receives an instruction to reheat/re-cool to the reference temperature (returnToRef operation) and it moves to ReturningToRef state. In ReturningToRef state, the air conditioner consumes energy to reach the reference temperature. When the reference temperature is reached, the air conditioner reports the event (refTempReached notification) and moves to the SteadyRef state. The air conditioner may be switched off by switchOff operation in any of the states GoingToRef, SteadyRef, GoingToEcon, SteadyEcon and ReturningToRef.

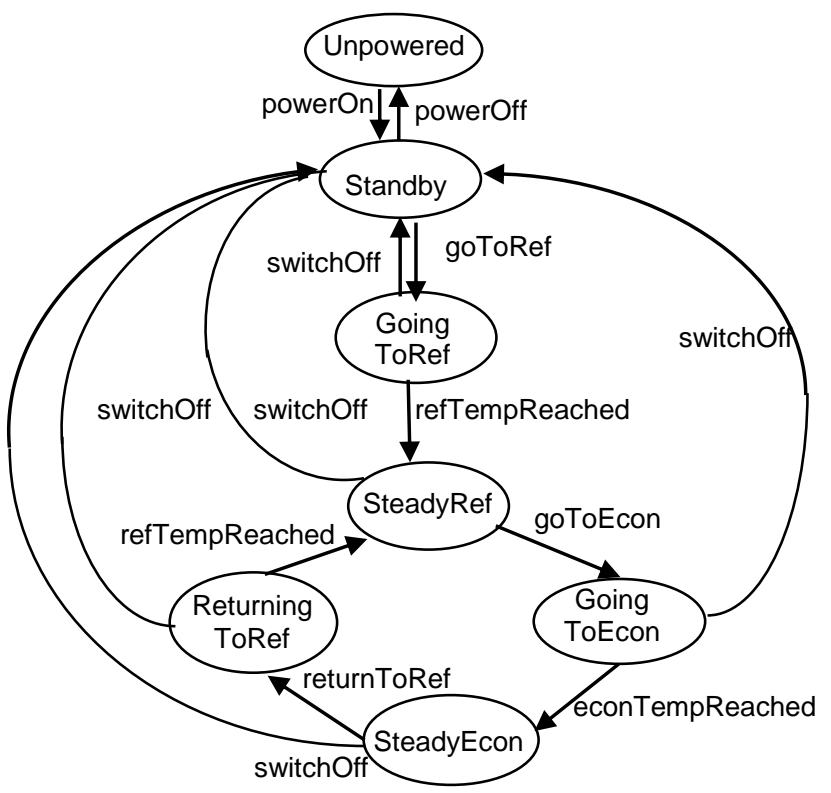

Fig. 1. State model of air conditioner

\subsection{Resource structure for power control support}

As to [16], M2M Service Capabilities:

- provide M2M functions that are to be shared by different applications;

- expose functions through a set of open interfaces;

- use core network functionalities;

- simplify and optimize application development and deployment through hiding of network specificities. 
M2M Service Capabilities may be implemented in the Network Service Capability Layer (NSCL) exposing functionally to network applications (NAs), and in the Device/Gateway Service Capability Layer (D/GSCL) exposing functionality to device/gateway applications (D/GAs). M2M service capabilities can be regarded as service bricks providing specific functions which may be reused in different M2M applications. The NSCL is operated by an M2M service provider.

The proposed generic functionality for remote management of home electrical appliances needs to be deployed both in the network as provided as homeApplianceControl NSCL and in the device (air conditioner) airconXXX DSCL. The exposed functions allows configuration of schedules for power control and control on energy consumption by home appliances according to predefined schedules. The DSCL discovers NSCL so as to perform registration. Let us assume that HomeEnergyMgmt is an NA that may be used for heating/cooling control of electrical appliances at home needs. In order to be remotely controlled, the respective device (air conditioner) needs to support to register with NSCL. A particular air conditioner XXX, which is installed, runs a powerControl DA which allows power control. The HomeEnergyMgmt NA registers to NSCL, and powerControl DA registers with DSCL.

Fig.2 shows the resource structure after registration and announcement of powerControl DA to the NSCL.

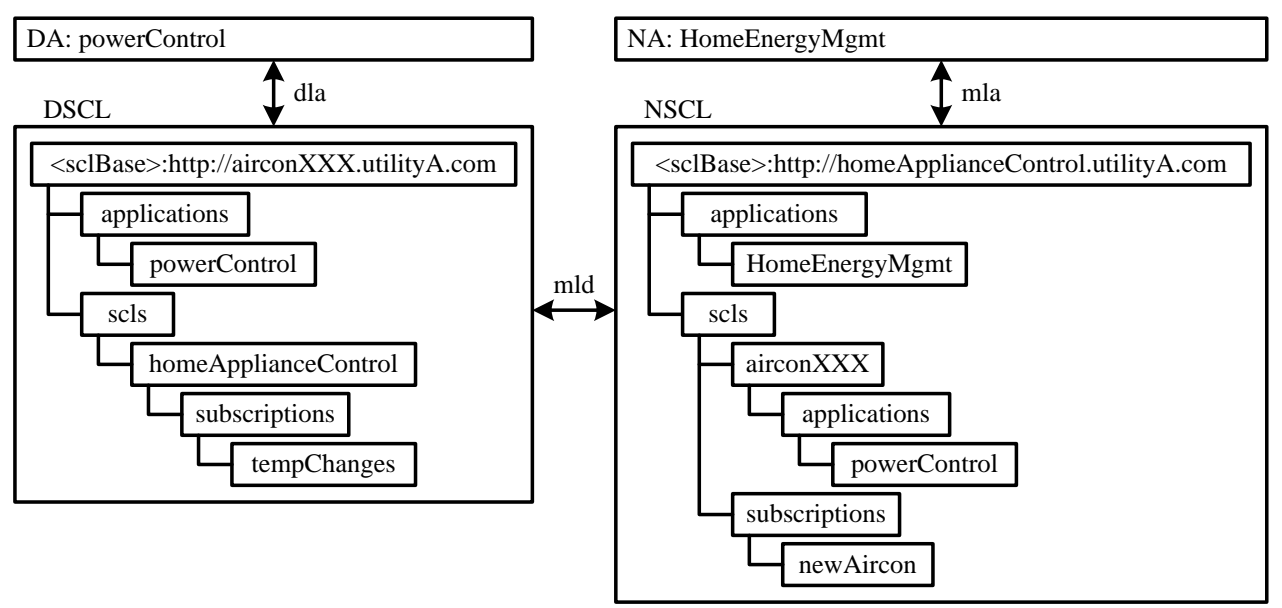

Fig. 2. Tree structure model of smart metering resources supporting power control of electrical appliances

The air conditioner owner may define family allowance aimed to save energy using the energy management application. Fig. 3 shows the resource structure for configuration management function of the power control scheme.

Management objects are created for each air conditioner to allow management functions. The seasonSchedule resource is defined as a management object resource which contains management data for configuration of air conditioner season schedules. It represents a structured management object containing up to four season schedules each defined as season resource. Daily schedules may be defined 
as sub-resources for each season. Different daily schedules may be defined for working days, weekends and holidays. Each daily schedule defines the reference temperature and the daily periods with demand-response management. Different daily periods may be defined when everybody is at home, outside and at nights when everybody sleeps. Each daily period is defined by it's begin time, end time and the tolerance assumptions for changes of home reference temperature.

NSCL

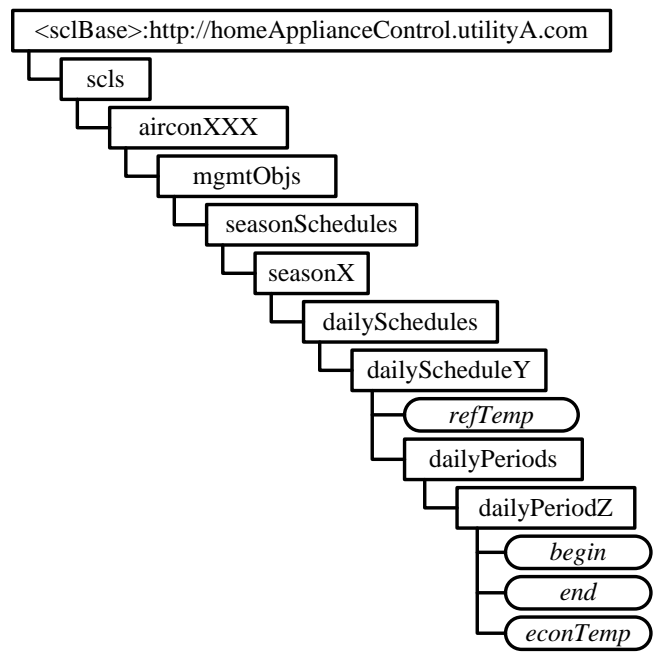

Fig. 3. Scheduling resource structure for power control

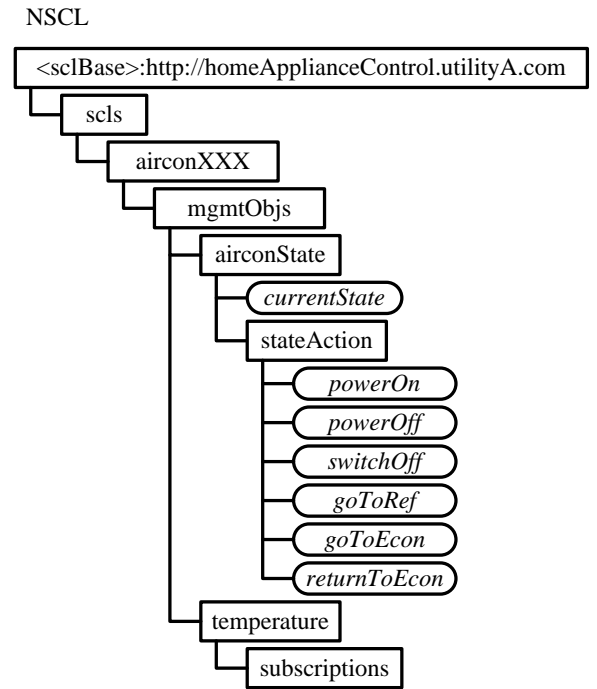

Fig. 4. Air conditioner state resource structure

The NA may handle the air conditioner as a resource by manipulating its states. Fig. 4 shows the pattern of the resource structure representing the air conditioner states. Common resource attributes like creation time, access rights, etc., may be found in [16]. In this paper we stress on domain-specific resources and attributes which can not be found in specifications.

\subsection{Web service interfaces for home appliance control}

We propose two web services that may be exposed to applications for home appliance control. The proposed web services interfaces expose functionality over the mla and mld interfaces.

The Schedule Management web service provides interfaces for remote configuration of heating/cooling control schedules. Table 1 summarizes the interfaces and operations supported by the Schedule Management web service.

The seasonSchedulesMgmt interface allows NAs to retrieve and update information associated with a seasonSchedules collection resource.

The seasonScheduleMgmt interface allows NAs to manage the creation, retrieval, update and deletion of information associated with particular $<$ seasonSchedule> resource representing season schedules.

The dailySchedulesMgmt interface allows NAs to retrieve and update information associated with a dailySchedules collection resource. 
The dailyScheduleMgmt interface allows NAs to manage the creation, retrieval, update and deletion of information associated with a particular $<$ dailySchedule $>$ resource representing daily schedule.

Table 1. Summary of Schedule Management web service interfaces

\begin{tabular}{|l|l|l|}
\hline \hline Interfaces & Supported operations & Implementation \\
\hline \hline \multirow{4}{*}{ seasonSchedulesMgmt } & updateSeasonSchedules & HTTP PUT \\
\cline { 2 - 3 } & retrieveSeasonSchedules & HTTP GET \\
\hline \hline \multirow{5}{*}{ seasonScheduleMgmt } & createSeason & HTTP POST \\
\cline { 2 - 3 } & retrieveSeason & HTTP GET \\
\cline { 2 - 3 } & updateSeason & HTTP PUT \\
\cline { 2 - 3 } & deleteSeason & HTTP DELETE \\
\hline \hline \multirow{5}{*}{ dailySchedulesMgmt } & updateDailySchedules & HTTP PUT \\
\cline { 2 - 3 } & retrieveDailySchedules & HTTP GET \\
\hline \hline dailyScheduleMgmt & createDailySchedule & HTTP POST \\
\cline { 2 - 3 } & retrieveDailySchedule & HTTP GET \\
\cline { 2 - 3 } & updateDailySchedule & HTTP PUT \\
\cline { 2 - 3 } & deleteDailySchedule & HTTP DELETE \\
\hline \hline \multirow{5}{*}{ dailyPeriodsMgmt } & updateDailySchedules & HTTP PUT \\
\cline { 2 - 3 } & retrieveDailySchedules & HTTP GET \\
\hline \hline \multirow{3}{*}{ dailyPeriodMgmt } & createDailyPeriod & HTTP POST \\
\cline { 2 - 3 } & retrieveDailyPeriod & HTTP GET \\
\cline { 2 - 3 } & updateDailyPeriod & HTTP DUT \\
\cline { 2 - 3 } & deleteDailyPeriod & \\
& &
\end{tabular}

The Appliance Power Control web service provides interfaces for manipulating the air conditioner state, which are summarized in Table 2.

Table 2. Summary of Appliance Power Control web service interfaces

\begin{tabular}{|c|c|c|}
\hline Interfaces & Supported operations & Implementation \\
\hline \multirow[t]{4}{*}{ AirconStateMgmt } & createState & HTTP POST \\
\hline & retrieveState & HTTP GET \\
\hline & modifyState & HTTP PUT \\
\hline & deleteState & HTTP DELETE \\
\hline \multirow[t]{3}{*}{ StateActionMgmt } & createStateAction & " HTTP POST \\
\hline & executeStateAction & HTTP PUT \\
\hline & deleteStateAction & HTTP DELETE \\
\hline \multirow[t]{4}{*}{ TemparatureMgmt } & createTemp & $\begin{array}{l}\text { HTTP POST } \\
\end{array}$ \\
\hline & retrieveTemp & HTTP GET \\
\hline & modifyTemp & HTTP PUT \\
\hline & deleteTemp & HTTP DELETE \\
\hline \multirow[t]{2}{*}{ "TempSubscMgmt } & "getTempSubs & $\begin{array}{l}\text { HTTP GET } \\
\end{array}$ \\
\hline & updateTempSubs & HTTP PUT \\
\hline \multirow[t]{4}{*}{ RefTempSub } & createRefTemp & HTTP POST \\
\hline & retrieveRefTemp & HTTP GET \\
\hline & modifyRefTemp & HTTP PUT \\
\hline & deleteRefTemp & HTTP DELETE \\
\hline \multirow[t]{2}{*}{ RefTempNotification } & refTemperature & HTTP PUT \\
\hline & tempError & HTTP PUT \\
\hline \multirow[t]{4}{*}{ EconTempSub } & createEconTemp & HTTP POST \\
\hline & retrieveEconTemp & HTTP GET \\
\hline & modifyEconTemp & HTTP PUT \\
\hline & deleteEconTemp & HTTP DELETE \\
\hline EconTempNotification & $\begin{array}{l}\text { econTemperature } \\
\text { tempError }\end{array}$ & $\begin{array}{l}\text { HTTP PUT } \\
\text { HTTP PUT }\end{array}$ \\
\hline
\end{tabular}


The dailyPeriodsMgmt interface allows NAs to retrieve and update information associated with a dailyPeriods collection resource. The dailyPeriodMgmt interface allows NAs to manage the creation, retrieval, update and deletion of information associated with particular $\langle$ dailyPeriod $\rangle$ resource.

\section{Prepaid smart metering}

\subsection{Identification of requirements to the support of prepaid smart metering}

Prepayment functionality requires real-time control of energy usage [21, 22]. It shall be possible to apply energy usage monitoring for the accumulated usage of energy by a consumer on per predefined daily periods. This capability is required for enforcing dynamic policy decisions based on the total energy usage in real time. The smart metering information system that uses energy usage monitoring for making dynamic policy decisions shall set and send the applicable thresholds to the smart meters for monitoring. The energy usage monitoring thresholds shall be based either on time or on volume. The smart metering information system may send both thresholds to the smart meters. The smart meter shall notify the smart metering information system when a threshold is reached and report the accumulated usage since the last report for energy usage monitoring. If both time and volume thresholds were provided to the smart meter, the accumulated energy usage since last report shall be reported when either the time or the volume thresholds are reached. Usage monitoring on smart meter level is active provided that certain conditions are met. For energy usage monitoring at smart meter level the applicable condition is a volume and/or time threshold has been provided and there is at least one rule activated for critical peak periods. Energy usage monitoring allows to connect a supply and to disconnect it after a predetermined consumption or certain time duration.

\subsection{Resource structure for prepaid functionality support}

The minimum revenue billing and functionality requirements for the residential single phase, self-contained meter types include among the others and up to four daily schedules, time of use plus dynamic tier for four seasons, real-time pricing tier, and load profile [22]. The utilities may use some or all of the defined functionality, as to their business model and/or customer regulatory requirements.

Following the requirements, we model as tree structures resources representing configuration of tariff schedules and management of prepaid profiles. Fig. 5 shows the tree model of the configurable data about schedules. The schedule information may include static schedules and dynamic tier. The static schedule contains information for up to 4 seasons, up do 4 daily schedules, and up to 4 daily periods. The dynamic tier allows definition of critical periods for energy consumption. Fig. 6 illustrates the resource structure extension for prepaid functionality.

The support of energy usage monitoring requires definition of managed objects that set usage monitoring trap events notification. Such events include reaching consumption/time thresholds. Trap events managed objects are defined to 
allow network applications to be notified about events of interest. Such managed objects are defined for each daily period, defined in each daily schedule in particular season schedule.

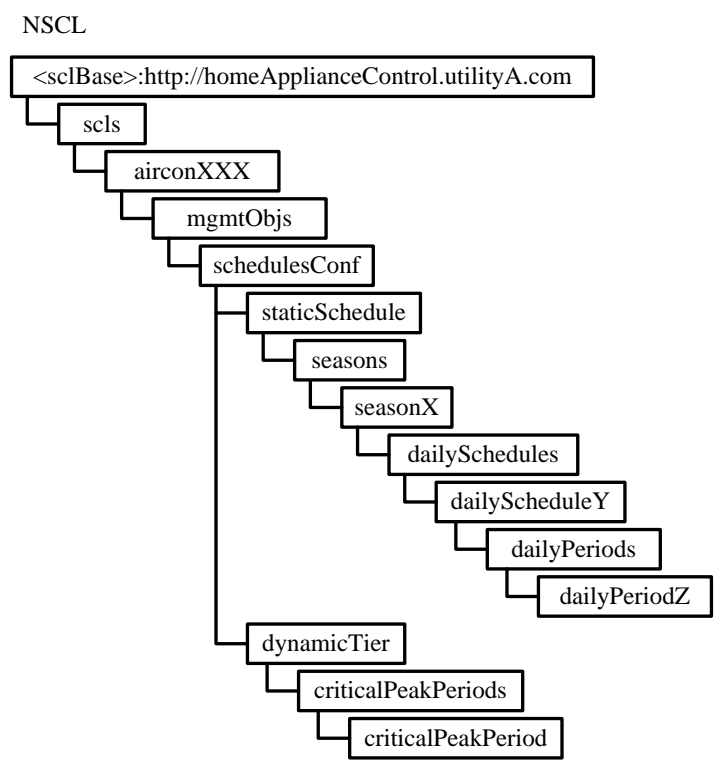

Fig. 5. Resource structure of the managed object for configuration of tariff schedules

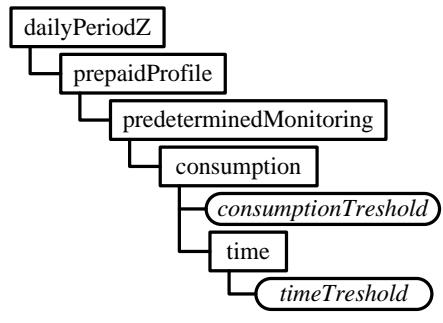

a) An extension of daily period resource for prepaid subscription

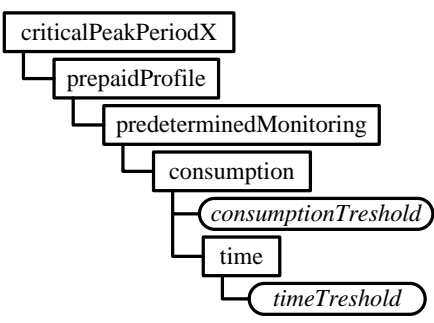

b) An extension of critical peak period resource for prepaid subscription

Fig. 6. Extensions with prepaid profiles

\subsection{Prepaid smart metering web service interfaces}

Web service interfaces for prepaid smart metering support functionality required to configure schedules for advanced tariffing of energy usage as well as functions for energy usage monitoring. An interface is defined for each resource. The supported interface operations allow resource creation and deletion, and update and retrieval of resource attributes. For interfaces representing collection-resource the operations allow only retrieval and update of information about resources.

\section{Discussions on approach applicability}

\subsection{Novelty of the proposed approach}

The idea of power control on residential appliances is not new. It captures the attention of many researchers [2, 4-8]. The existing works provide efficient solutions which face specific application requirements. This has led to heterogeneity where interoperability is very limited. In order to cope with fragmented market, current research on $\mathrm{M} 2 \mathrm{M}$ communications is aimed at development of horizontal service platforms which provide capabilities that can be reused across different applications. Key factor is the separating applications from service capabilities and network capabilities (e.g., policy). 
We present an approach to provide service capabilities for energy management. The approach includes data modelling and web service interface definition. Data models explicitly determine the structure of data exchange between M2M applications residing in the device and in the network. The logic behind the use of data models is that the applications share common view on data structures which extends interoperability. Our data models are domain-specific and define structured resources representing controllable appliance states, schedules for applying power control, energy usage monitoring and prepaid energy consumption. The proposed models are in conformance with recommended by the standards resource tree structures. Definition of web services is based on outlined generic functionality which supports appliance power control, schedule management and prepaid smart metering. The generic functions are exposed to different applications by web service interface operations. Identified operations allow resource manipulation by four primitives which can be implemented by HTTP requests.

\subsection{Estimation of the home appliance power control potential}

The impact estimation, achievable by the use of RESTful web services, is based on well-adopted methodology regarding the thermal characteristics of households. Two cases are compared: a) M2M-based schedule of two high-temperature $\left(21{ }^{\circ} \mathrm{C}\right)$ intervals, namely 6:30-9:00 $\mathrm{AM}$ and 5:30-10:00 PM, while the reference temperature is kept lower $\left(20^{\circ} \mathrm{C}\right)$ for the rest of the day; b) constant reference $\left(22^{\circ} \mathrm{C}\right)$.

The average room is assumed to be $30 \mathrm{~m}^{3}$, and windows of $4.6 \mathrm{~m}^{2}$, equipped with $5 \mathrm{~kW}$ device $\left(75 \%\right.$ efficiency, hysteresis of $\left.1{ }^{\circ} \mathrm{C}\right)$. The transmittances of walls and windows are about 1 and $5 \mathrm{~W} /\left(\mathrm{m}^{2} . \mathrm{K}\right)$ respectively. The cooling follows the Newton's law and the room thermal mass $\mathrm{C}_{\mathrm{T}}$ is $10^{7} \mathrm{~J} / \mathrm{K}$.

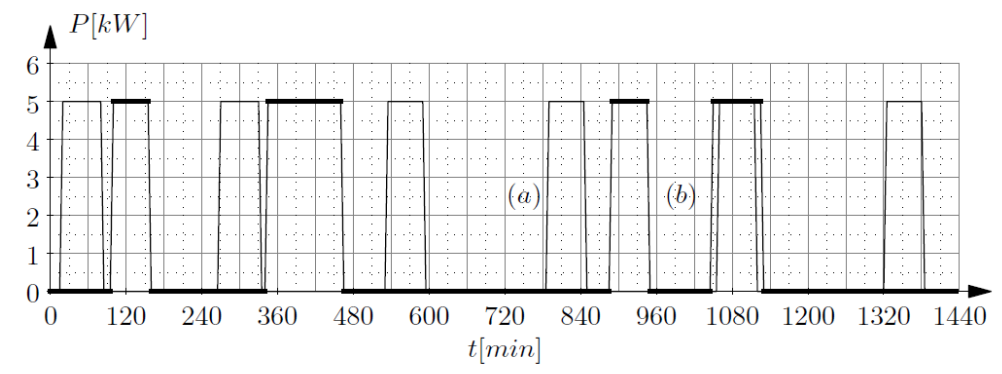

Fig. 7. Power consumption: traditional (a); IoT-based schedule (b)

In Fig. 7, it is shown power consumption versus time when the traditional control is applied and when IoT-based schedule is adopted. It might be noticed that the 'discomfort' for the households of keeping two degrees lower temperature by the introduction of RESTful web services-based schedule causes consumption reduction i.e. saving and change in the pattern like a time-shift.

To estimate the "bigger picture" it is assumed that for the city it is known: total number of households is 600,000; percentage of households using air conditioners is 5\%; expected RESTful web services-based control technology acceptance is 
$20 \%$. Having the single-household effect and with the late assumptions it is trivial that the daily and weekly effects would be 25 and 125 MW.h respectively. As a reference it may be pointed out that the Earth Day campaign has lead to a saving of about 56 MW.h.

\section{Conclusion}

Advanced smart metering is aimed at improvement of energy end-use efficiency and thus contributing to reduction of energy consumption and mitigation of green gasses emissions. The usage of M2M technology for smart metering allows communications with and direct control on individual devices within home/buildings and supporting flexible tariffing and payment systems.

The paper presents a structural approach to the design of RESTful web services for home appliance control with prepaid functionality. The relevant information is modelled by tree structures and may be shared between different applications. Data mediation functions of RESTful web services are exposed to applications through addressing resources. The suggested resource structures describe different types of smart metering resources that may be manipulated by data-life-cycle related actions. The web service interfaces implement specific actions on resources by four primitives used to create/delete a resource and to retrieve/update the content of the resource. The potential of the proposed RESTful web services for home appliance power control is estimated for a typical household and the large scale effect is highlighted.

RESTful web services increase scalability because every communication is stateless, i.e. requests are handled independently and the resource state may be transferred by HTTP proxies and browsers to the clients. Further RESTful web services are more appropriate to constrained devices such as smart meters and electrical appliances which can be read or configured and even stream data to the outside world. The proposed approach is simple and easy to use which greatly reduces the efforts needed to develop M2M applications.

\section{References}

1. K i n g, N., P. J e s s e n. Smart Metering Systems and Data Sharing: Why Getting a Smart Meter Should Also Mean Getting Strong Information Privacy Controls to Manage Data Sharing. International Journal of Low and Information Technology, Vol. 22, 2014, No 3, pp. 215-253.

2. Bharanialankar, S., C. ManikandaBabu. Intelligent Home Appliance Status Intimation Control and System Using GSM. - International Journal of Advanced Research in Computer Science and Software Engineering, Vol. 4, 2014, No 4, pp. 554-556.

3. K a marudin, M., M. Yus of. Low Cost Smart Home Automation via Microsoft Speech Recognition. - International Journal of Engineering \& Computer Science, Vol. 13, 2013, pp. 6-11.

4. Y a n, M., H. S h i. Smart Living Using Bluetooth Based Android Smartphone. - International Journal of Wireless \& Mobile Networks, Vol. 5, 2013, pp. 65-72.

5. Vasak, M., A. Starcic, A. Martincevic. Model Predictive Control of Heating and Cooling in a Family House. International Convention MIPRO, 2011, pp.739-743. 
6. B e ghi, A., L. Cecchinato, M. Rampazzo. Thermal and Comfort Control for Radiant Heating/Cooling Systems. - In: Proc. of IEEE International Conference on Control Applications (CCA), 2011, pp. 258-263.

7. A b a a k k a i l, R., M. Orozco, A. E 1 S a d dik. Home Energy Saving for Heating/Cooling System by Distributed Intelligent Energy Controller. - In: Proc. of IEEE International Instrumentation and Measurement Technology Conference (I2MTC), 2012, pp. 604-609.

8. L i n, G. Optimal Energy Management in Microgrids with Integrated Multi-Zone Heating/Cooling Control. Master Thesis, 2014.

MASc.pdf. https://macsphere.mcmaster.ca/bitstream/11375/16052/1/Lin_Guotao_2014September_

9. B ingol, O., K. Ta sde len, Z. Ke skin, Y. Koc at u rk. Web-Based Smart Home Automation: PLC-Controlled Implementation. - Acta Polytechnica Hungarica, Vol. 11, 2014, No 3, pp. 51-63.

10. Raikar, S., S. Majigoudar, K. Rithushree, R. Rohith, K. Venkates h. Prepaid Power Billing Using Adaptive Meter. - International Journal of Advanced Research in Electrical, Electronics and Instrumentation Engineering, Vol. 3, 2014, No 6, pp. 9912-9920.

11. Ellenki, S., S. Reddy, C. Srikanth. An Advanced Smart Energy Metering System for Developing Countries. - International Journal of Scientific Research and Education, Vol. 2, 2014, No 1, pp. 242-258.

12. S hu kla, A., S. S a x en a, S. S in gh, S. J a in. Embedded System Based Prepaid Electricity Metering \& Billing. - International Journal of Emerging Trends in Electrical and Electronics (IJETEE), Vol. 2, 2013, No 4, pp. 40-43.

13. Pras ad, M., Y. Kiran. Prepayment of Electricity Units with Theft Controlling System. International Journal of Research Studies in Science, Engineering and Technology (IJRSSET), Vol. 1, 2014, No 4, pp. 42-48.

14. Moh a m mad, N., A. B arua, M. Arafat. A Smart Prepaid Energy Metering System to Control Electricity Theft. - International Conference on Power, Energy and Control (ICPEC), 2013, pp. 562-565.

15. V a 11 u r u, S. Design and Assemble of Low Cost Prepaid Smart Card Energy. - International Journal on Electrical Engineering and Informatics, Vol. 6, 2014, No 1, pp. 65-73.

16. ETSI TS 102690 Machine-to-Machine Communications (M2M); Functional Architecture. V1.1.1, 2011.

17. B e lq a s mi, F. RESTful Web Services for Service Provisioning in Next-Generation Networks: A Survey. - IEEE Communications Magazine, Vol. 49, 2011, pp. 66-73.

18. E $1 \mathrm{~m}$ ang ou sh, A., T. Magedanz, A. B lot ny, N. B 1 u m. Design of RESTful APIs for M2M Services. - In: Proc. of International Conference on Intelligence in Next Generation Networks (ICIN), 2012, pp. 50-56.

19. M u th u l a k s m i, A., R. L a th a. The SOAP Based Mechanism for Home Environment Using Web Services. - Electrical \& Computer Engineering: An International Journal (ECIJ), Vol. 3, 2014, No 2, pp. 53-60.

20. T a k a t s u ka, H., S. S a i ki, S. M at s u m o t o, M. N a k a m u ra. A Rule-Based Framework for Managing Context-Aware Services Based on Heterogeneous and Distributed Web Services. - In: Proc. of IEEE/ACIS International Conference on Software Engineering, Artificial Intelligence, Networking and Parallel/Distributed Computing (SNPD), 2014, pp. 1-6.

21. ETSI TR 102691 Machine-to-Machine Communications (M2M); Smart Metering Use Cases, V1.1.1, 2010.

22. EEI-AEIC-UTC. Smart Meters and Smart Meter Systems: A Metering Industry Perspective, White Paper. A Joint Project of the EEI and AEIC Meter Committees, 2011. 